\title{
Inclusion Population Evolution in Ti-alloyed Al-killed Steel during Secondary Steelmaking Process
}

\author{
Enno ZINNGREBE, ${ }^{1)}$ Corrie VAN HOEK, ${ }^{1)}$ Henk VISSER, ${ }^{1)}$ Albert WESTENDORP() and In-Ho JUNG ${ }^{3)}$ \\ 1) Tata Steel Research Development \& Technology, P.O. Box 10,000, 1970 CA IJmuiden, The Netherlands. E-mail: \\ enno.zinngrebe@tatasteel.com $\quad$ 2) Tata Steel Strip Products Mainland Europe, P.O. Box 10,000, 1970 CA IJmuiden, The \\ Netherlands. $\quad 3)$ Department of Mining and Materials Engineering, McGill University, 3610 University Street, Montreal, \\ QC H3A 2B2, Canada. E-mail: in-ho.jung@mcgill.ca
}

(Received on May 19, 2011; accepted on August 22, 2011)

\begin{abstract}
This paper presents a new approach towards the evolution of non-metallic inclusion (NMI) populations in Ti-alloyed Al-killed steels, based on an extensive inclusion analysis campaign at Tata Steel Europe, IJmuiden Works. Automated SEM techniques were used to characterize the inclusion populations in 120 steel samples taken from nine heats out of two casting series of this steel grade. As NMI in Ti-alloyed Alkilled steels are overwhelmingly dominated by chemically simple $\mathrm{Al}_{2} \mathrm{O}_{3}$, most of the process relevant information lies in the analysis of particle size distribution during the secondary steelmaking process. The population density function (PDF) concept was applied, for the first time, to the characterization of inclusion size distributions sampled from secondary steelmaking practice. Two size distribution forms predominate in the entire dataset: i) Lognormal size distributions associated with active nucleation and growth of alumina (deoxidation and reoxidation), indicating net transfer of matter between NMI and solutes in liquid steel and ii) Power-law size distributions, associated with an inclusion population in chemical equilibrium with the melt and subject to collision/breakup processes controlling the distributions. Based on inclusion PDF observations, it is found that the size distribution of alumina inclusions suspended in steel melt, after equilibration and effective float out of large inclusions, tends to approach a Reference Distribution of powerlaw type function $\left(f(r)=a \cdot r^{-3.5}\right)$ that appears to be a fundamental feature of the alumina-steel system. This Reference Distribution can guide efforts to improve and engineer inclusion populations for a better controlled steel product.
\end{abstract}

KEY WORDS: non-metallic inclusion; inclusion population density function; inclusion size distribution; secondary steelmaking.

\section{Introduction}

One of the main challenges in the production of high quality steels is to control the population of non-metallic inclusions (NMI) over the whole process route in the steel plant. The presence of NMI's can compromise the properties of the final steel product and also negatively affect the refining process by depositing in the feeding systems of the continuous caster. This deposited layer limits the steel flow through the feeding system and can break off, leading to productivity and/or surface quality problems. For this reason, the interaction between existing NMI in liquid steel and the various installations is in the center of attention for secondary refining process. Recently, details of the inclusion formation of Ti-alloyed Al-killed steel $\operatorname{grades}^{1-5)}$ and its physical and chemical development during reoxidation are receiving considerable attention. From these studies, it is being recognized that inclusions in the $\mathrm{Al}-\mathrm{Ti}-\mathrm{Fe}-\mathrm{O}$ system may react sensitively on the details of the secondary steelmaking treatment, such as the oxygen balance achieved.

In the last decade sophisticated tools like automated SEM-EDS techniques have become available enabling the examination of the chemical and physical properties of large amounts of inclusions. Data conversion methods to obtain size distributions of the analyzed inclusion are further assisting the study of the evolution of inclusion in steel processing. Usually, the inclusion size distributions are given in the form of histograms using pre-defined bin-sizes. However, as discussed by Van Ende et al.," the Population Density Functions (PDF) provides a practical and user-independent form of the summary of the inclusion size distribution. From theoretical numerical calculations ${ }^{7,8)}$ of inclusion PDF's, it is known that an inclusion size distribution can change with time, from a lognormal shape distribution at the early stage after nucleation, to a power-law shape distribution at a later mature state after growth, agglomeration and separation. Thus, inclusion size distribution data (PDF) can provide insights on the inclusion formation event/timing and on the inclusion formation mechanism.

The present study focuses explicitly on the evolution of the inclusion populations along the entire secondary steelmaking route from the moment of deoxidation in the ladle up to the cast steel in the mould. Extensive sampling trials were carried out during the production process of two sequences of Ti-alloyed Al-killed steel grade in the Basic Oxygen Steel plant No.2 of Tata Steel Strip Products Main- 
land Europe. The automated SEM technique (Automated Inclusion Analysis or AIA) in conjunction with a data conversion method of raw data to inclusion PDF was used in the present study to characterize the inclusions in large size plant samples. The evolution of inclusion PDF will be discussed with the events in the secondary steelmaking process.

\section{Industrial Test \& Sampling Procedure}

\subsection{Chemistry of Steel and Processing Condition}

Samplings were carried out for a Ti-alloyed Al-killed steel grade. The secondary steelmaking process for this steel is carried out by the following steps:

i) Tapping of about 320 ton of steel from a converter into a ladle lined with several different refractories depending on the position.

ii) Transfer to RH-OB degasser, where after the decarbonization process the $\mathrm{Mn}$ content is adjusted by adding Fe-Mn. After 10-15 min, the molten steel is deoxidized by addition of granular $\mathrm{Al}$, followed by $\mathrm{Fe}-\mathrm{Ti}$ alloy addition after 2-3 minutes to adjust Ti concentration. Post-treatment stirring by purging Ar gas.

iii) Transfer to the rotating turret of the caster and standby for casting to tundish.

iv) After opening the sliding gate of a ladle, steel is cast into a two-strand 65 ton tundish.

v) Steel is cast through two-port submerged entry nozzle (SEN) into the moulds of two thick slab casting strands controlled by a sliding gate system.

\subsection{Sampling of Steels}

In order to characterize the evolution of inclusions of this steel, a major effort was expended into extensive samplings of two casting sequences of Ti-alloyed Al-killed steel. Of the first casting sequence A, samples from three initial heats (A1 to A3) were examined. The main data of this study are formed by the samples of all six heats (B1 to B6) of a second casting sequence $B$.

The total secondary steelmaking processing time of each steel heat from deoxidation at the RH-OB degasser to beginning of casting was typically about $30-50$ minutes. To examine the evolution of inclusions directly after deoxidation at the RH-OB degasser, the early heats of each series (heats A1, A2 and B1) were sampled directly after the Al addition, using standard lollipop samplers roughly in a minute interval. This resulted in 5 to 7 time series samples of the respective three heats up to completion of the RH-OB treatment. Subsequent heats were routinely sampled: one sample after completion of RH-OB treatment. The subsequent samplings between RH-OB and caster consisted of lollipop samples before and after steel stirring at the stirring station. After transport to the caster, Ar blow-suck samples were obtained from the top of the ladle at the turret before opening the ladle. After ladle opening, steels were sampled at three locations in the tundish by Ar blow-suck sampling: at the direct vicinity of the ladle shroud, and above both strands. Simultaneously mould samples were obtained using standard lollipop mould samplers. For the initial heats of each series (A1 and B1), the tundish and mould samplings were performed about every $5 \mathrm{~min}$ until $30 \mathrm{~min}$. The other heats were sampled at 5-10 min and $30 \mathrm{~min}$ after opening the ladle.

In total, 120 steel samples of both series (39 from series A, and 81 from series B) were prepared and analyzed for this study.

\subsection{Inclusion Analyses and Data Classification Tech- niques}

All steel samples were analyzed for their bulk steel chemical compositions at the in-house analytical facilities at IJmuiden works, Tata Steel Europe. The bulk chemical analyses were done by spark OES and combustion analysis (the latter for $\mathrm{C}, \mathrm{N}, \mathrm{O}$ ). The compositions achieved in this cast included about $400 \mathrm{ppm}$ Ti and $290 \mathrm{ppm} \mathrm{Al}$.

\subsubsection{Automated Inclusion Analysis (AIA) System}

After bulk chemical analysis, the NMI in the samples was analyzed by AIA system at Tata Steel's Ceramic Research Center (CRC) at IJmuiden works. For the inclusion analysis, small steel blocks directly under the spots of the spark-OES analysis were cut out from the steel samples. Then, the blocks were mounted in conductive resin and polished for inclusion analysis allowing a $\sim 5 \mathrm{~mm} \times 5 \mathrm{~mm}\left(25.3 \times 10^{-6} \mathrm{~m}^{2}\right)$ analyzable cross section.

The AIA was performed using a Jeol JSM5900LV SEM operating at $15 \mathrm{kV}$ and equipped with a ThermoElectron NORAN System Six (NSS) microanalytical system. A customized Feature Sizing Setup was used, with the following settings: $250 \times$ magnification, $0.247 \mu \mathrm{m}$ pixel size, and a $4 \times 4$ pixel minimum size corresponding to a lower limit of $0.75 \mu \mathrm{m}^{2}$ for inclusion detection. Objects (inclusions or other features) were detected on Back Scatter Electron (BSE) mode image frames using automatic thresholding of the image grey level, and their EDS spectrum acquired using beam rastering mode. Objects were accumulated up to a numerical limit of 2001 objects, or to reaching the full $5 \times$ $5 \mathrm{~mm}$ observational surface, whichever came first. Generally, the 2001 raw objects limit was reached only in the deoxidation samples with their high alumina cluster content. A pre-acquired matrix (steel) EDS spectrum was subtracted from every object spectrum and only the residual spectrum was quantified, allowing reliable analysis of inclusion objects much smaller than the electron beam excitation volume. Residual matrix-subtracted spectra were quantified for F, Na, Mg, Al, Si, P, S, Cl, K, Ca, Ti, V, Cr, Mn, Cu, Zn, $\mathrm{Zr}, \mathrm{Nb}$ and in some runs also $\mathrm{Ni}$. All object residual spectra and analysis results were recorded together with a predefined set of morphological features including cross sectional area, perimeter, and location on the automated-stage reference frame.

\subsubsection{Correction of AIA Spectrum}

The primary AIA output is a set of objects, not all of which are true inclusions, as any analyzed steel surface also presents a number of artifacts (e.g. scratches, holes, dust and other surface deposits). Therefore, inclusion datasets must be cleaned to remove such non-inclusion objects. For this purpose an in-house developed filtering routine was used, based on the raw chemical compositions analyzed for each object. If an object recognized by the SEM is merely a topographical irregularity of the steel (such as scratch and hole), then the residual EDS quantified spectrum is a noise spectrum, yielding comparable spectral intensity across the entire elemental range. On the other hand, true NMI in steel can contain significant amounts of only a restricted and known set of elements. In this Ti-alloyed steel grade, for example, the restricted (expected) set of elements are $\mathrm{Al}$, $\mathrm{Mg}, \mathrm{Si}, \mathrm{Ca}, \mathrm{S}, \mathrm{Mn}$ and $\mathrm{Ti}$ (and N, which is undetectable by EDS). The wide range of elements in the original quantification allows therefore discrimination of noise objects, and 
part of the total spectral intensity usually belongs to the expected element group from real non-metallic inclusions. After application of the filter based on the expected elements, some types of mineral dust and deposits (e.g. $\mathrm{MgO} /$ $\mathrm{CaSiO}_{3}$ dust, $\mathrm{CaCO}_{3}$ from ambient humidity) have to be removed by manual post-processing. On average, more than $40 \%$ of all originally detected objects had to be removed as artifacts and contaminations in the overall data cleansing process. This removal rate of artifacts and contaminations is strongly dependent on the development of clean sample handling procedure; the recovery rate of above $75 \%$ was achieved in the final stage of this study. After data cleansing process in this study, the smallest inclusion data set contained 114 and the largest contained 1791 individual inclusions from the sample of $5 \times 5 \mathrm{~mm}$ (on average 664 inclusions per dataset).

After data cleansing, the resultant inclusion files were processed as follows. To assess the bulk inclusion chemistry, two chemical population characteristics were calculated. The average bulk composition of an inclusion assemblage was calculated from a phase renormalization of the individual inclusions. It was found that an overwhelming fraction of nearly all inclusions could be expressed as a linear combination of the compounds $\mathrm{MnS}, \mathrm{Ti}(\mathrm{O}, \mathrm{N})_{\mathrm{x}}, \mathrm{MgAl}_{2} \mathrm{O}_{4}$, $\mathrm{MnAl}_{2} \mathrm{O}_{4}$, and $\mathrm{Al}_{2} \mathrm{O}_{3}$. Individual inclusions were recast into amounts of these phases, which after factoring in phase densities, allowed the summation over each individual's contribution to derive the average bulk composition of the inclusion. Furthermore, based on the inclusion density, the non-dissolved elements associated with NMI can be calculated. In the authors' experience, the amount of nondissolved oxygen calculated from the inclusion density shows the same order of magnitude value from conventional combustion method measurement. However, in the present study, oxygen amount calculations based on AIA technique were not used, because even this precision is considered not good enough to estimate meaningful soluble/insoluble oxygen in steel samples.

\subsubsection{Inclusion Population Density Function (PDF)}

A large part of the information of the AIA data lies in physical-morphological features, which can be transformed to the particle size distribution. Particle Size Distributions (PSD) or Crystal Size Distributions (CSD) is a well-established subject in chemical engineering and associated fields, ${ }^{9,10)}$ and this methodology is adopted in the present study to treat the steel NMI PSD directly taken from the AIA data. The size distribution data are not expressed as traditional histograms (frequency $f$ per chosen size bin $\Delta l$ ), as the numerical values of such histograms strongly depend on arbitrary observer's choices for bin size setting. Instead, inclusion size distributions are expressed as Population Density Functions (PDF), where the population density $n(l)$ of particles of a certain size $l$ is defined as the change in the frequency $\Delta f$ of an adjoining size bin as this bin is expanded across the size of interest:

$$
n(l)=\Delta f / \Delta l
$$

The PDF can be written for lengths $n(l)$, cross sectional areas $n(a)$, or volumes $n(v)$, depending on the measurement method employed. SEM based inclusion analysis is a cross sectional area observation method and as such it is subject to stereological limitations, i.e. the presence of non-central sections through inclusions that prohibit a direct recalcula- tion of the analysis results as particle size frequencies. In order to overcome this, a stereological correction routine has been applied using, CSD Corrections v. $1.36,{ }^{11,12)}$ which was developed to convert $2 \mathrm{D}$ cross sectional data into reconstructed 3D particle size populations. This correction routine employs a regular stripping, modified by a chosen shape parameter (relative axis lengths and roundness of particles) as well as potential sample anisotropy. In the case of alumina inclusions in liquid steel, the samples can be regarded as isotropic. The outputs of this correction procedure are modeled 3D particle size distribution, its population density function, and modeled cross sectional area of inclusions. The stereologically corrected PDFs were calculated for all inclusion populations using this method and plotted against the equivalent inclusion diameter, a standard plotting procedure on which phenomenologically and genetically distinct distribution functions can most easily be discriminated. A detailed discussion of the PDF representation and the procedure to calculate PDF plots are given in previous studies ${ }^{13,14}$ ) as well as the paper by Van Ende et $a .^{6)}$

\section{Results and Discussion}

\subsection{Al Deoxidation/Ti Alloying Process at RH-OB Degasser}

Average chemistry of non-metallic inclusions

The variations of chemistry and size distribution of inclusions during the deoxidation process were assessed based on series of samplings for three heats (A1 and A2 as well as B1). As mentioned earlier, Mn alloying was carried out before $\mathrm{Al}$ deoxidation in $\mathrm{RH}-\mathrm{OB}$ degasser, which produced $\mathrm{Mn}$-rich liquid $(\mathrm{Mn}, \mathrm{Fe})(\mathrm{O}, \mathrm{S})$ inclusions. Because the steel contains $\mathrm{S}(\sim 70$ wt. ppm), it is expected to form $\mathrm{MnS}$ or Mn oxysulfide during Mn alloying. The AIA analyses showed that the average molar ratio of $\mathrm{S} / \mathrm{Mn}$ in the inclusions are about 1 for some samples (heat B1) and less then 0.05 for other samples (heat A2). As Fe was not measured in the AIA analyses, the $\mathrm{Mn} / \mathrm{Fe}$ ratios are not known. In the case of heat B1, the calculated amount of S associated with $\mathrm{MnS}$ inclusion after Mn alloying was about 13 wt. ppm, which dropped to about 0.2 wt. ppm after Al deoxidation. In the case of heat $\mathrm{A} 2,(\mathrm{Mn}, \mathrm{Fe}) \mathrm{O}$ inclusions dominated in steel before $\mathrm{Al}$ deoxidation, and inclusion associated $\mathrm{Mn}$ concentration dropped from $601 \mathrm{wt}$. ppm to less then $1 \mathrm{wt}$. ppm by Al deoxidation.

After $\mathrm{Al}$ deoxidation, the inclusion compositions are vastly dominated by $\mathrm{Al}_{2} \mathrm{O}_{3}$. This is expected from well-known Al deoxidation reactions:

$$
\begin{array}{r}
2 \underline{\mathrm{Al}}+3 \underline{\mathrm{O}}=\mathrm{Al}_{2} \mathrm{O}_{3} \ldots \ldots \ldots . . . \\
3 \mathrm{MnO}+2 \underline{\mathrm{Al}}=\mathrm{Al}_{2} \mathrm{O}_{3}+3 \underline{\mathrm{Mn}} .
\end{array}
$$

With the subsequent Ti alloying process, the chemical composition of inclusions can be varied toward Ti containing alumina inclusions due to the following chemical reactions:

$$
\begin{aligned}
& 5 \mathrm{Al}_{2} \mathrm{O}_{3}+9 \underline{\mathrm{Ti}}=3 \mathrm{Ti}_{3} \mathrm{O}_{5}+10 \underline{\mathrm{Al}} \\
& x \mathrm{Al}_{2} \mathrm{O}_{3}+(3 y+2 z) \underline{\mathrm{O}}+(2 \mathrm{y}+\mathrm{z}) \underline{\mathrm{Ti}}=
\end{aligned}
$$

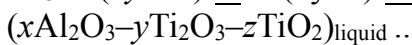

The chemical reactions are highly dependent on the oxidation state and local concentration of Ti in melt. The homogenization of melt composition in the RH-OB degasser process after $\mathrm{Al}$ deoxidation and $\mathrm{Ti}$ alloying takes several 
minutes. Thus, the local composition of steels just after Ti alloying in RH-OB process is quite inhomogeneous. Figure 1 shows the relationship between steel composition and inclusion composition in the samples after Ti addition. The results of heats $\mathrm{A} 1, \mathrm{~A} 2$ and $\mathrm{B} 1$ were obtained at between $10 \mathrm{~s}$ to $316 \mathrm{~s}$ after $\mathrm{Al}$ deoxidation. The results from other heats are from samples at about $270 \mathrm{~s}$ to $310 \mathrm{~s}$ after Al deoxidation. It should be noted that the Ti additions were carried out at between $120 \mathrm{~s}$ and $180 \mathrm{~s}$ after $\mathrm{Al}$ deoxidation and the final $\mathrm{Ti} / \mathrm{Al}$ ratio in the present Ti-alloyed Al-killed steel is about 1 . Clearly, the molar $\mathrm{Ti} / \mathrm{Al}$ ratio in non-metallic inclusions shows the strong correlation with the dissolved Ti/Al ratio in molten steels, consistent with a curved form as expected from a reaction constant expression for Eq. (4). It should be also noted that even after Ti alloying, $80-96 \%$ of individual inclusions were found to be pure $\mathrm{Al}_{2} \mathrm{O}_{3}$ in most of the samples.

Correction of inclusion size distribution due to secondary TiN inclusions forming during quenching

The chemical distribution of inclusions after Al deoxidation and Ti alloying shows an interesting feature. Figure 2 shows the molar $\mathrm{Ti} /(\mathrm{Ti}+\mathrm{Al} / 2)$ ratio (which is denoted as $\mathrm{Ti} \#$

(a)

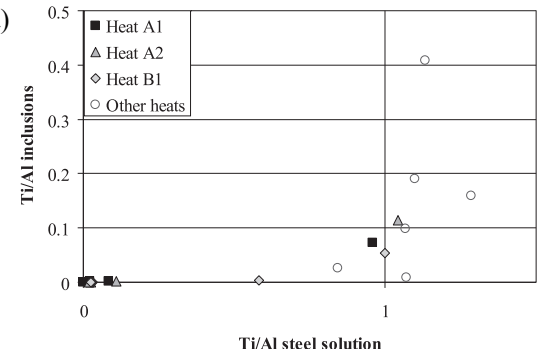

(b)

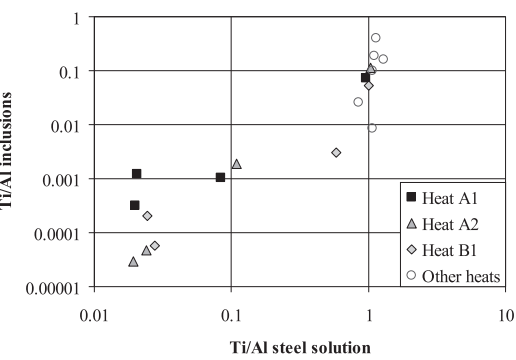

Fig. 1. Apparent correlation of $\mathrm{Ti} / \mathrm{Al}$ (atomic) ratios between the inclusion assemblages and the steel melts during deoxidation in the two casting series of Ti-alloyed steel. (a) Linear scale and (b) logarithm scale. Closed symbols are time series analyses of three heats (A1, A2 and B2) spanning a time between 0 and 5 minutes after deoxidation (each starts at low $\mathrm{Ti} / \mathrm{Al}$, ends at high $\mathrm{Ti} / \mathrm{Al}$ ). Open circles are values at the end of $\mathrm{RH}-\mathrm{OH}$ in six other heats. in this study) of inclusions compared to their size distribution in heats $\mathrm{A} 1$ and $\mathrm{A} 2$ after about $300 \mathrm{~s}$ of $\mathrm{Al}$ deoxidation. In the case of heat A2, a large amount of inclusions less than $2 \mu \mathrm{m}$ in diameter are located in Ti\# $>0.9$. EDS observations confirmed that the inclusions of Ti\# $>0.8$ are mostly TiN and $\mathrm{TiN} / \mathrm{Al}_{2} \mathrm{O}_{3}$ inclusions. The typical morphology of such TiN-bearing inclusions is presented in Fig. 3(a). The TiN phase is believed to be produced during the quenching of samples, and thus it should not be counted in the inclusion analysis for molten steel condition. In the present study, therefore, the inclusions with Ti\# $>0.8$ were not considered in any further inclusion analyses with PDF concept. This correction can substantially influence the accuracy of the PDF for small inclusions (less than $2 \mu \mathrm{m}$ ). The very variable occurrence of this TiN precipitation in the earliest stages of alloying contributes to the scatter of the average $\mathrm{Ti} / \mathrm{Al}$ of the bulk inclusion populations in Fig. 1, which shows uncorrected data.

\section{Existence of liquid $\mathrm{Ti}-\mathrm{Al}-\mathrm{O}$ oxide inclusions}

In the case of heat $\mathrm{A} 1$, the amount of inclusions in the range of Ti\# $=0.4-0.8$ are quite significant (Fig. 2). The typical morphology of the inclusions in this range is shown in Fig. 3(b). The Ti-rich and Al-rich phases are encapsulated in a thin but continuous shell of pure $\mathrm{Al}_{2} \mathrm{O}_{3}$. Similar complex inclusions were also reported by Park et l. $^{4)}$ and Doo

(a)

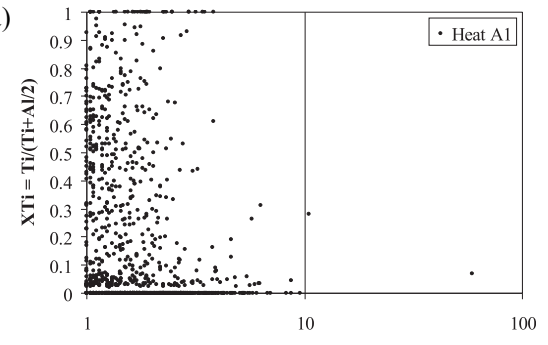

(b)

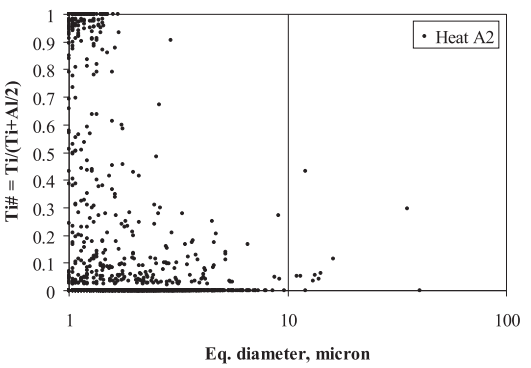

Fig. 2. Inclusion size - composition plots at the end of RH-OB for two heats from series A (A1 and A2). Ti\# is molar ratio of $\mathrm{Ti} /(\mathrm{Ti}+(\mathrm{Al} / 2))$ and equivalent diameter (Eq. diameter) is in $\mu \mathrm{m}$. Total number of inclusions are 1677 and 1527 for heat $\mathrm{A} 1$ and $\mathrm{A} 2$, respectively.

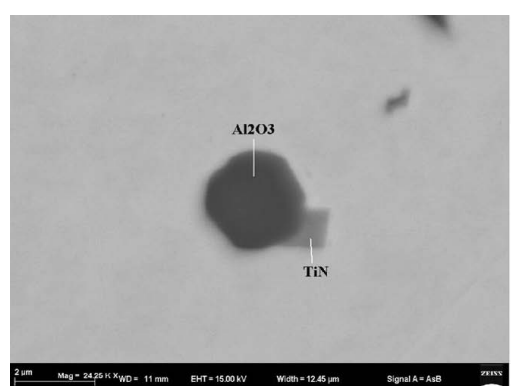

(a)

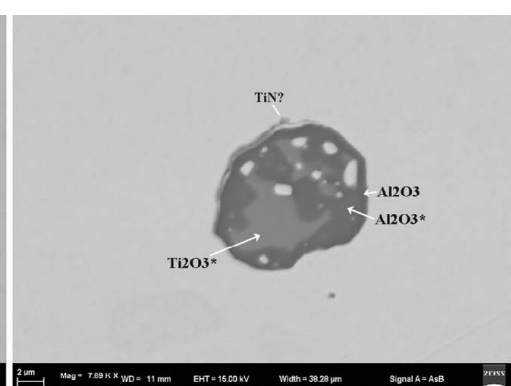

(b)

Fig. 3. Typical inclusions observed after $\mathrm{RH}-\mathrm{OB}$. (a) $\mathrm{TiN}+\mathrm{Al}_{2} \mathrm{O}_{3}$ inclusions formed during solidification as secondary inclusion $(\mathrm{Ti} \#=0.22)$ and $(\mathrm{b}) \mathrm{Al}-\mathrm{Ti}-\mathrm{O}$ complex inclusions in a shell of pure $\mathrm{Al}_{2} \mathrm{O}_{3}\left(\mathrm{Ti}_{2} \mathrm{O}_{3} *\right.$ and $\mathrm{Al}_{2} \mathrm{O}_{3}$ * represent the phases containing a considerable amount of $\mathrm{Al}_{2} \mathrm{O}_{3}$ and $\mathrm{Ti}_{2} \mathrm{O}_{3}$ respectively, $\mathrm{Ti} \#=0.5$. As noted in Eq. (4), however, the stoichiometry of $\mathrm{Ti}$ oxide can be closer to $\mathrm{Ti}_{3} \mathrm{O}_{5}$ ). 
et $a l .{ }^{5)}$ from a sample taken at the RH-OB degasser after Ti addition, where they were believed to be in liquid state (at least inner side). The overall chemical composition (Ti\#) of the inner side of the inclusion is about $0.66-0.76$ as defined above, $\mathrm{Ti} /(\mathrm{Ti}+\mathrm{Al} / 2)$, which is in good agreement with the present results. According to the calculated phase diagram of the Al-Ti-O oxide system in equilibration with Al-deoxidized molten steel (Jung et al. ${ }^{15)}$ ) using the FactSage ${ }^{\mathrm{TM}}$ optimized thermodynamic database, ${ }^{16}$ a possible liquid phase of the $\mathrm{Al}-\mathrm{Ti}-\mathrm{O}$ with $\mathrm{Ti} \#=0.7-0.75$ can be calculated in saturation with $\mathrm{Al}_{2} \mathrm{O}_{3}$ at $1600^{\circ} \mathrm{C}$, which is also similar to the present observation. A small amount of $\mathrm{ZrO}_{2}$ (several wt. \%) was also observed in the inclusion, consistent with an origin from $\mathrm{Zr}$ containing ferro-Ti alloys.

\section{Population Density Functions (PDF) after Deoxidation}

The analysis of particle size distributions in the preferred population density function (PDF) form is complicated by the presence of very strong clustering of inclusions in the post-deoxidation samples. This is illustrated in Fig. 4(a) which represents the $2 \mathrm{D}$ cross-section of the examined sample of heat A2 at about 4.5 min after Al deoxidation. As can be seen, there are several clusters of inclusions. The clusters appearing as hundreds of individual NMI clustered in a small areal region are in fact 2D sections through complex $3 \mathrm{D}$ dendritically networked complexes of inclusions. In general, the distance between clusters is in the order of $\mathrm{mm}$. Although strictly speaking the observed sample area is not fully representative for the abundance of clusters as such, we found that the analysis area is sufficient to represent the general feature of individual inclusions in secondary steelmaking. As clusters are self-similar (see below), under- or over-representation of cluster inclusions in the observed area only causes an absolute magnitude shift of the apparent PDFs (see Fig. 5).

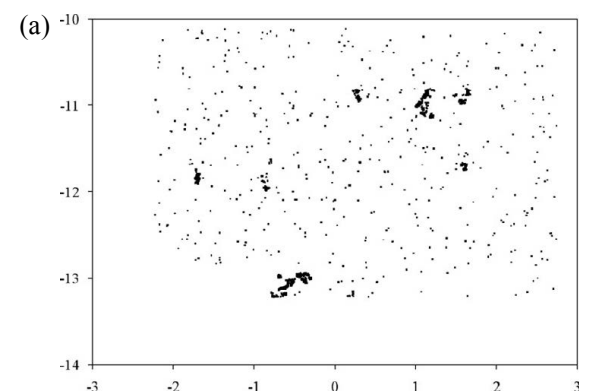

(b)

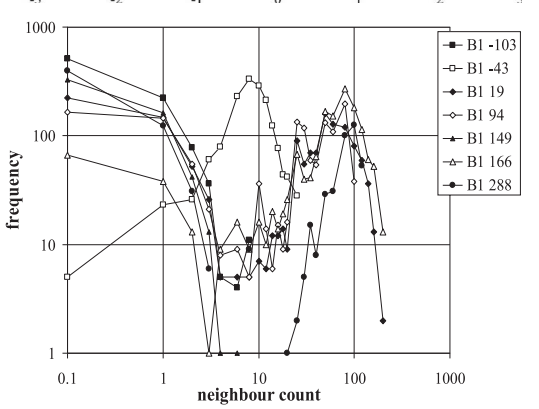

Fig. 4. Overview of inclusion size distribution. (a) Heat A2 sampled at $273 \mathrm{sec}$ after deoxidation (total number of inclusion = $1527 ; 6 \times 4 \mathrm{~mm}^{2}$ ). Each point is location of a single individual, but real inclusion sizes are far less than point sizes on this figure. (b) Variation of inclusion distribution for heat $\mathrm{B} 1$ with neighbour count within $\mathrm{a} \pm 50 \mu \mathrm{m}$ search mask. Inclusions with no neighbours within this mask are plotted as having " 0.1 " neighbors. Numbers in the legend refer to the time in seconds from nominal $\mathrm{Al}$ addition.
In order to clearly distinguish the clusters from the inclusions occurring as isolated individuals (in the present study, this is called a 'matrix inclusion'), the frequency of inclusion neighbor counts is plotted in Fig. 4(b). Considering that the distance between matrix inclusions is larger than $50 \mu \mathrm{m}$, for the cluster-containing samples, we counted for each individual inclusion cross section the number of neighbors (separate inclusion cross sections) within $\mathrm{a} \pm 50 \mu \mathrm{m}$ box. The frequency of such neighbor counts is shown in Fig. 4(b). The inclusions are clearly divided into two groups: (i) matrix inclusions with only less than 10 neighboring inclusions within the search box and (ii) cluster inclusions with more than several tens of neighboring inclusions. Accordingly, we used a value of 10 (corresponding to a local inclusion frequency of $0.001 \mu \mathrm{m}^{-2}$ ) to split the datasets into subsets of inclusions being part of matrix and clusters respectively. Matrix and cluster inclusion populations were then analyzed separately. In the case of sample B-43, the trend is completely different from others. In this steel, Mn was intentionally alloyed before Al-killing to observe the influence of the formation of (MnO-FeO) liquid oxide inclusion. As liquid ( $\mathrm{MnO}-\mathrm{FeO}$ ) inclusion is emulsified liquid droplets in liquid steel, they do not tend to cluster like solid $\mathrm{Al}_{2} \mathrm{O}_{3}$ inclusion, and therefore the trend of the size distribution of sample B-43 is different from others.

Population Density Functions (PDF) of cluster inclusions and matrix inclusions for three pre-deoxidation datasets, and three post-deoxidation time series available from heats A1, A2 and B1 are plotted in Figs. 5 and 6 respectively. Strikingly, the results of cluster inclusions show lognormal type PDF and on the other hand the results of matrix inclusions show almost linear power-law type PDF.

The markedly upwards-convex curve part of the PDF of cluster inclusions (Fig. 5) can be fitted well as lognormal size distributions: ${ }^{13,14)}$

$$
f(r)=\left(f_{0} / r\right)\left[\frac{\exp \left(-\frac{(\ln r-a)^{2}}{2 b^{2}}\right)}{\sqrt{2 \pi \cdot b^{2}}}\right]
$$

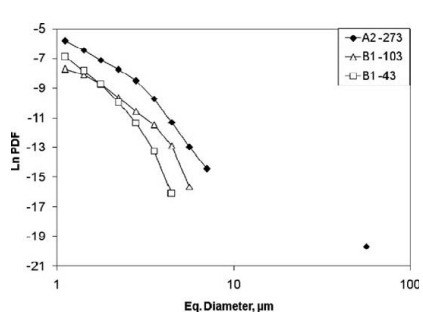

(a)

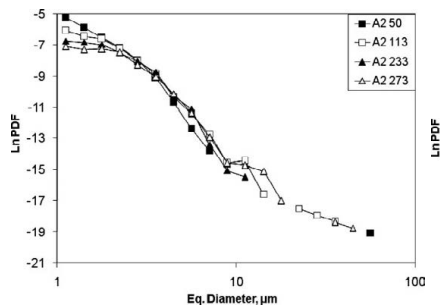

(c)

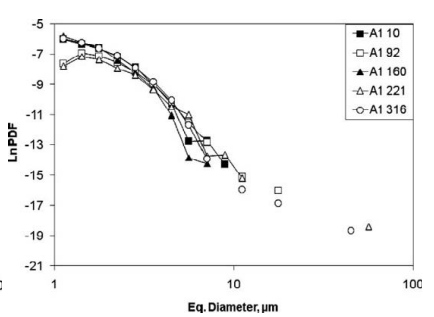

(b)

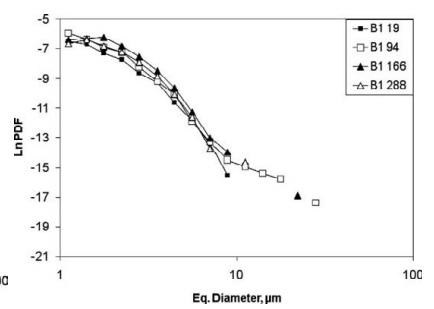

(d)
Fig. 5. Particle size distributions in form of inclusion PDF plots for the cluster inclusions before and after deoxidation process. (a) Inclusion PDF before deoxidation and (b)-(d) after deoxidation. Numbers in the legend refer to the time in seconds from nominal $\mathrm{Al}$ addition. 


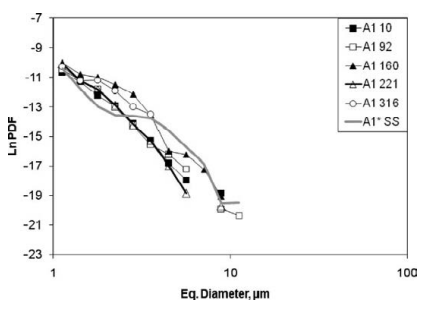

(a)

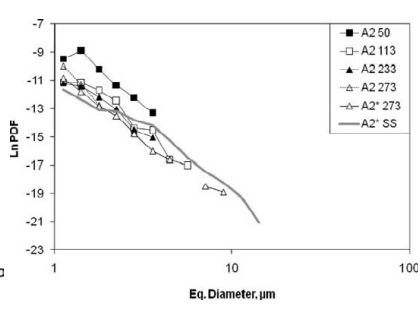

(b)

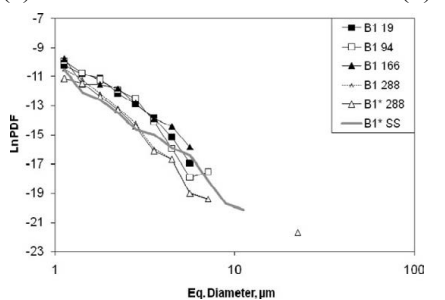

(c)

Fig. 6. Particle size distributions in form of inclusion PDF plots for the individual (matrix) inclusions before and after deoxidation process. Numbers in the legend refer to the time in seconds from nominal $\mathrm{Al}$ addition. SS stands for the results for the same heat approximately $15 \mathrm{~min}$ later at the stirring station.

wherein $f(r)$ is the PDF of inclusions, a (scale parameter) and $b$ (shape parameter) are shape parameters controlling peak position and wideness of the lognormal, while a scaling factor $f_{0}$ sets the general particle abundance level. The trace of such a distribution on a doubly logarithmical PDF plots as those employed herein is parabolic. For example, the fitting parameters for such lognormals on the observed datasets for A1 (221 sec): $f_{0}=0.00187, a=0.279, b=0.616$, and B1 (288 sec): $f_{0}=0.00242, a=0.518, b=0.433$.

It has to be noted that nearly all the apparent lognormal curves given in Fig. 5 have their maximum at or near to the lower size limit of the present analyses $(1 \mu \mathrm{m})$. This restricts the quality of fitting. That is, $a$ parameter is only weakly constrained in a fit nearly entirely based on only one side of a lognormal. Conversely, the $b$ parameter is much better constrained.

The populations of small inclusions (less than $2 \mu \mathrm{m}$ ) in all samples are decreasing with time, which is a typical feature of the Ostwald ripening. Although a significant decrease of small inclusions below $1 \mu \mathrm{m}$ can be expected, such inclusions were not detectable in the AIA setup used here. In terms of the mathematical function in Eq. (6), it would be expressed as a time decrease of the $b$ parameter (sharpening of the distribution and lowering of its variance ${ }^{13)}$ ). Although it is rather scattered due to the inhomogeneity of melt chemistry, the general trend of $b$ for all samples shows the decrease of $b$ with time, which results from the Ostwald ripening of inclusions. A better quantification of the PDF of such cluster inclusions, as possible with FEG-SEM resolution of sub-micrometric individuals, would make it possible to directly calculate the Ostwald ripening rates of alumina in liquid steels.

The PDFs of the matrix inclusions in Fig. 6 show a linear decrease within the scatter of the experimental data. Although a few of the PDFs do display some curvature, none of them are close to the regular log-normal shape like the PDF of cluster inclusions in Fig. 5. This PDF of matrix inclusions corresponds to another mathematic functional form that is widely recognized in particulate engineering studies, the power-law, or fractal, distribution: ${ }^{13,14)}$

$$
f(r)=a \cdot r^{-\mathrm{b}}
$$

where the exponent $b$ is the slope of the line; and $a$ is a scaling pre-factor setting the overall abundance of the particle distribution.

The different PDF behaviour of cluster inclusions and matrix inclusions can be explained by the different growth mechanisms of the inclusions in molten steel. In the case of cluster inclusions, the inclusion inside of the cluster has no chance to collide with other individual inclusions in molten steel. The cluster as a whole can collide with individual matrix inclusions or other clusters, or it can be broken down by itself. These events can influence only some inclusions in the outer layer of cluster but not inclusions in the inner part of the cluster. Thus, the only growth mechanism for inner cluster inclusions is Ostwald ripening, which tends to preserve original nucleation-derived lognormal $\mathrm{PDF}^{18,19)}$ as shown in Fig. 5. On the other hand, the individual matrix inclusions can grow both based on Ostwald ripening and collision. The Ostwald ripening can occur only shortly after nucleation when small particles are located in short distance (less than $10 \mu \mathrm{m}$ ). After this time period the main growth mechanism is collision growth, which leads to a power-law PDF as shown in Fig. 6.

\subsection{Post-degassing Treatment: Stirring Station}

In this section, the changes in steel chemistry and inclusion PDF in Ti-alloyed steel between the end of RH-OB degasser treatment (including deoxidation and major alloying) and ladle opening are investigated. This segment comprised significant time (30 to 50 minutes) in the two series $\mathrm{A}$ and $\mathrm{B}$, with Ar purging at the stirring station as the main action. Data from this interval of the heat histories is thus suitable to assess the extended-time evolution of the inclusions formed by the deoxidation in $\mathrm{RH}-\mathrm{OB}$ degasser, as well as to find out whether the generally assumed inclusioncleaning effect of Ar purging at a stirring station is supported by actual inclusion population data.

\section{Change of chemical composition of molten steel}

The changes of the dissolved $\mathrm{Al}$ composition in steels for all series of heats B with processing time are plotted in Fig. 7. In the plots, the deoxidation event is set to be the reference of zero time. In general, the concentration of $\mathrm{Al}$ decreases with processing time. Certainly, it is found that dissolved $\mathrm{Al}$ drops during the stirring process. This drop is quite noticeable for heat B5, still noticeable for heats B1 and B2, but less significant in the case of heats B4 and B6 and very small for heat B3. The drop of dissolved Al during processing at the stirring station can be related to the reoxidation of steel by incidental open eye formation or other sources. For example, in the case of steel $\mathrm{B} 5$, the reoxidation behaviour can be witnessed by the inclusion PDF analyses as shown below.

\section{Particle Density Function (PDF) after Ar stirring}

As discussed above, a TiN phase can be precipitated as a secondary inclusion during the sampling process. Because this can have an influence on the PDF, all inclusions with $\mathrm{Ti} /(\mathrm{Ti}+\mathrm{Al} / 2)>0.8$ were discarded in post-degasser PDF analyses in the present study. It is found that there are almost no cluster inclusions in the steel sample in this stage, so it is unnecessary to distinguish between cluster and matrix inclusions.

The inclusion populations in heats B3 and B5 between degasser and caster are presented in Fig. 8. Samplings of T1 

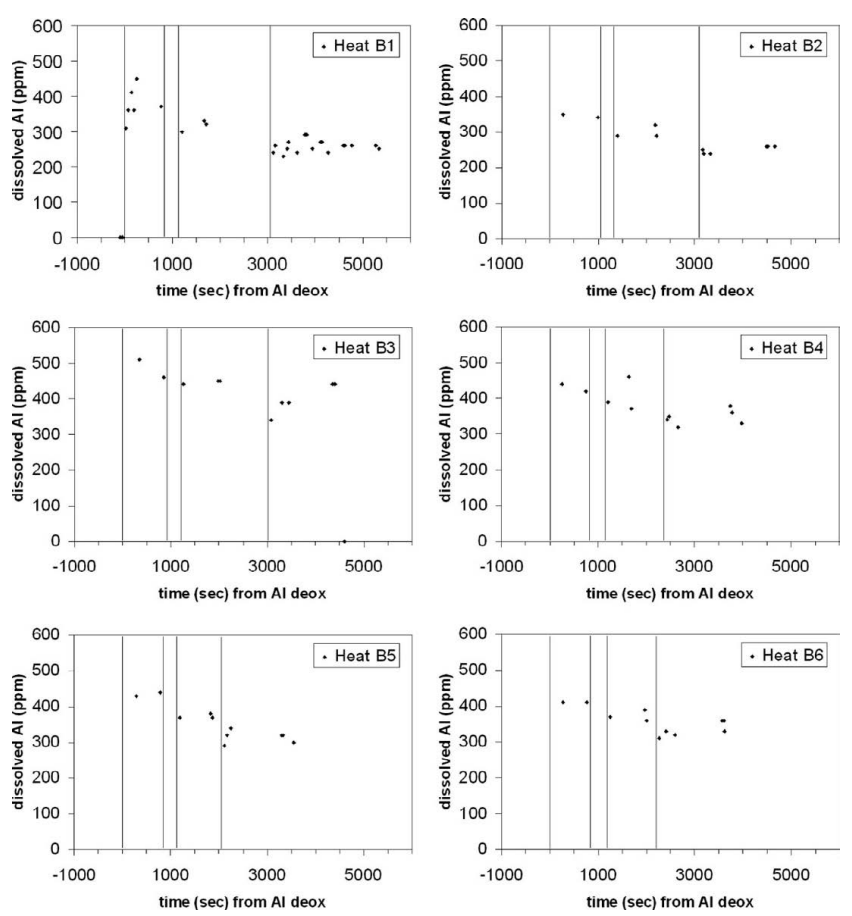

Fig. 7. Variation of dissolved Al content in the six heats of series B with secondary steelmaking process. All plots have four vertical lines denoting: Al addition, beginning of stirring, end of stirring, and beginning of casting.

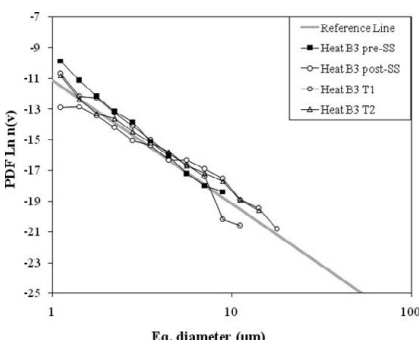

(a)

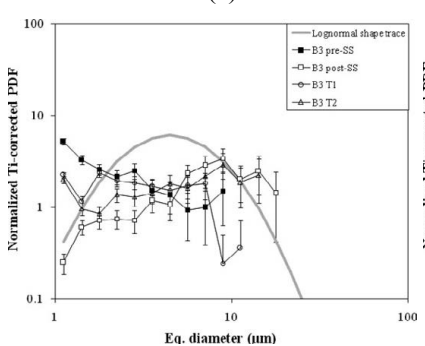

(c)

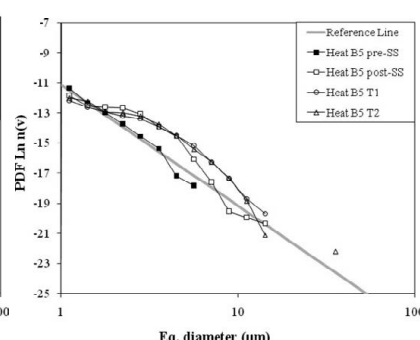

(b)

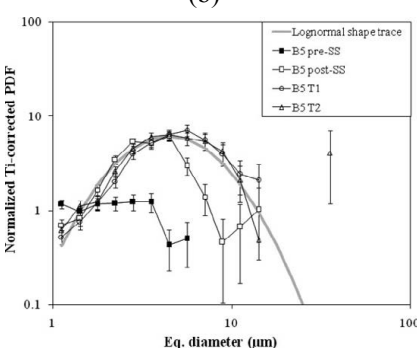

(d)
Fig. 8. Inclusion population density function (PDF) from the heat B3 and B5 between degasser and caster. (a) and (b) are original PDF, and (c) and (d) are normalized to the Reference Distribution (see Eq. (8)). Pre-SS and post-SS represent the samples taken before and after stirring station, respectively. T1 and T2 represent the sample doublet taken on the rotating turret of the caster before onset of casting. A power law distribution is plotted as Reference Line in the graphs (Eq. (8)).

and $\mathrm{T} 2$ represent the samples taken at the rotating turret of caster before casting. As it is apparent from a cursory inspection, the inclusion PDF curves for all pre-stirring station samples (at the arrival of stirring station before $\mathrm{Ar}$ stirring) fall into linear lines. If the steel chemistry and deoxidation process are the same for all B series heats, the inclusions PDF should theoretically be all the same. In fact, the PDF of all pre-stirring samples are very similar. Based on the average of all pre-stirring sample's PDFs, the linear base-line, approximating a power-law distribution, was derived and the line is also inserted to the plots in Figs. 8(a) and $8(\mathrm{~b})$ :

$$
f(r)=1.5 \times 10^{-5} \cdot r^{-3.5}
$$

where $f(r)$ is the population density function, and $r$ is the equivalent diameter of the inclusions. Lognormal-shaped deviations of inclusion PDFs from this base-line can be related to the oxidation (re-oxidation) behavior during the Ar stirring.

\section{Indication of Reoxidation by Ar stirring}

Most of the inclusion PDFs of series of B at stirring station have a very narrow band of distributions with an approximately linear behaviour, extending the PDF of prestirring condition. However, the inclusion PDFs of heat B5, for example, largely deviated from the base-line. In the case of steel heat B3, the deviation is very small and all the PDFs after stirring are still showing linear behaviour. Other heats such as B1, B2, B4 and B6 (which are not presented in the figure) showed small deviations from base line but were not as noticeable as heat B5.

The deviation of steel B5 from base-line line is an upward convex lognormal function, which is typically shown in the early stage of inclusion formation (the PDF is mainly dominated by nucleation and chemical growth; prior to collision growth stage). As also mentioned earlier in Fig. 7, a significant drop of dissolved $\mathrm{Al}$ was also recorded during stirring station treatment for the heat B5. The combination of these two observations leads to the conclusion that possible reoxidation happened during Ar stirring, most likely by the incidental formation of open-eye of ladle slag. The PDFs of newly nucleated $\mathrm{Al}_{2} \mathrm{O}_{3}$ inclusions takes a while to be straightened as linear line. In the other cases the smaller curved deviations in PDFs after Ar stirring seem to indicate a smaller degree of reoxidation.

In order to see the deviations clearly, the PDFs can be replotted normalized onto the base-line in Eq. (8). For example, the normalized PDFs for heats B3 and B5 are plotted in Figs. 8(c) and 8(d). In the case of the inclusions in heat B3, the normalized PDFs are scattered but horizontally distributed. On the other hand, the PDFs of heat B5 after stirring clearly show the lognormal hump shape. It can be also observed in Fig. 8 that stirring apparently reduced the number frequencies of small inclusions considerably and increased the amount of larger inclusions (for example, see the PDF of inclusions of $\sim 1 \mu \mathrm{m}$ in both heats B3 and B5). Heat B5 clearly shows evidence of renewed substantial precipitation of alumina during stirring: the post-stirring PDF with lognormal shape is explained by active crystallization. The amount of increase in alumina inclusions was calculated from the PDFs to be $\sim 160$ volume-ppm over the stirring stage and an additional 115 volume-ppm after stirring up to the turret. This corresponds to $\sim 70 \mathrm{ppm}$ of dissolved $\underline{\mathrm{Al}}$ transfer into the inclusion population, which is in good agreement with the change in chemical bulk steel analysis in Fig. 7.

The results in Fig. 8 tell that in fact the pre-stirring populations have in most cases significantly more linear size distributions than those found after stirring, and later after certain transit time on the rotating turret. These later populations tend to have higher actual number densities (and therefore, higher quantitative NMI amounts) than the pre- 
stirring population. Only heat B3, which shows the lowest apparent [Al] drop (20 ppm, close to analytical uncertainty) over stirring of the entire series, does not show this trend. On the other hand, heat B5, which shows the highest [Al] drops with $70 \mathrm{ppm}$, has a perfectly curved PDF shape at equivalent diameters $>1.5 \mu \mathrm{m}$ in all post-stirring samples even though the pre-stirring PDF was almost linear. Comparing this behavior with the observation at the RH-OB degasser that precipitation-formed alumina (the clusters) tends to lognormal size distributions, leads to the conclusion that the increase in inclusion amounts and change in distribution shape observed after stirring is caused by newly precipitated alumina. The occurrence of such undesirable processing effects can be detected both qualitatively, and evaluated quantitatively, with the methodology described here.

\subsection{Inclusion Population Behavior during Casting}

The casting operations of series A and B in this study involved a tundish filling volume (V) of 65 tons and a throughput (Q) of 6.5-8.0 ton/min, yielding a replenishment frequency of $\mathrm{Q} / \mathrm{V}=0.1-0.12 \mathrm{~min}^{-1}$. The change of chemistry of heats $\mathrm{A} 1$ and $\mathrm{B} 1$ with casting time was monitored. Inclusion size evolutions of the heats A1 and B1 (initial heats of each series) were investigated by up to 7 sets of triplet samples.

\section{Change of chemical composition of molten steel}

The change of the chemistry of heat B1 is presented in Fig. 9. The [Al] drop for heat A1 from ladle to tundish was about $50 \mathrm{ppm}$ and then little compositional change of $\mathrm{Al}$ was found between tundish and mould sampling spots. In contrast, the [Al] drop of heat B1 between ladle and tundish was about $70 \mathrm{ppm}$ and there was also a significant loss of [Al] and [Ti] between tundish and mould. Interestingly, the change of $[\mathrm{N}]$ in heat $\mathrm{B} 1$ clearly shows the inverse trend of [Al], which suggests that the reoxidation of steels by air happened during both the ladle/tundish and tundish/mould transitions. The concentration of $[\underline{\mathrm{N}}]$ in heat $\mathrm{B} 1$ at tundish and mould decreases with casting time. The same trends were observed for heat A1, the first series of A.

$\underline{\text { Population Density Function (PDF) during casting process }}$ Inclusion population changes during the casting process

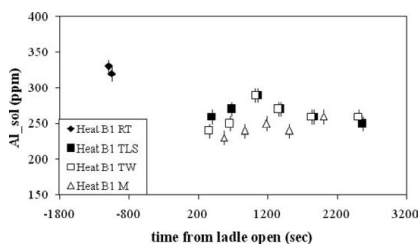

(a)

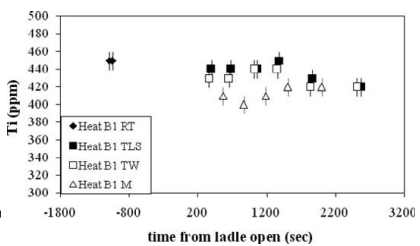

(b)

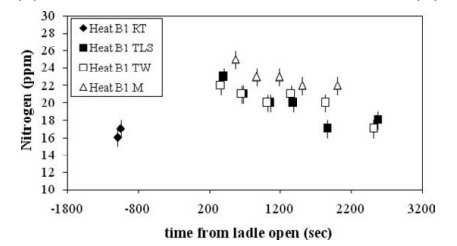

(c)

Fig. 9. Compositional evolution of the steel melt during casting for heat B1. (a) [Al], (b) [Ti] and (c) [N]. The sampling positions are indicated in the legend: $\mathrm{SS}=$ stirring station (after stirring); RT $=$ rotating turret of caster; TLS = tundish near to ladle shroud; TW = tundish above well; $\mathrm{M}=$ mould. are given in Fig. 10. All inclusion populations roughly follow a band along the power-law reference line distribution of Eq. (8). In detail, however, the inclusion density at each sampling spot in both heats decreases over the casting time, and the lowest population density values are always found towards the end of the casting. The only exception of this trend is the ladle shroud sampling position (Fig. 10(a)) for heat B1, where the last sample taken close to emptying of that ladle shows a very large increase in inclusion numbers (most strongly, inclusions with larger sizes). Inspection of this last sample showed the presence of alumina clusters similar in appearance to deoxidation alumina cluster inclusions, which is thought to be formed due to the vortex entrainment of oxidized slag at low steel level in the ladle. This slag entrainment can lead to strong reoxidation of the last steel cast. Except this accidentally reoxidized sample, all samples show that the inclusion densities at tundish and mould are decreasing with casting time. Besides the continuous decrease in inclusion amount over time, it should be noted that most of the initial PDFs at each sampling spot show pronounced curvature, but it becomes linear with time. Although the results are not presented here, the same trends were observed for heat A1.

The evolution of the inclusion PDF of heat B1 (for which the most detailed time sequence is available) for each inclusion size in tundish and mould with casting time is plotted in Fig. 11. The number of inclusions for each size is loglinearly decreasing until 20-30 min after opening ladle shroud and become almost constant after then until the end of casting of the heat $\mathrm{B} 1$. The same results were obtained for heat A1. This trend can be explained as follows. In the early stage of pouring the first heat of series from ladle to tundish, a relatively large amount of reoxidation can happen with the consumption of $\mathrm{Al}$ (see in Fig. 9) because the fresh tundish can have oxygen sources such as newly installed refractories and air occupying an empty tundish, as well as the ladle well filler sand which can participate in the reoxidation. Thus, the steel in the tundish just after beginning of opening of the ladle may be relatively highly reoxidized resulting in the formation of new inclusions. Then, the reox-

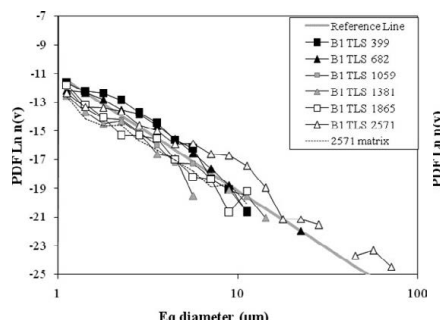

(a)

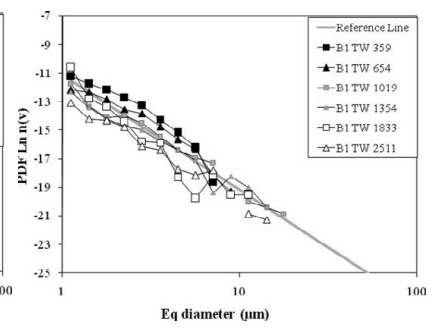

(b)

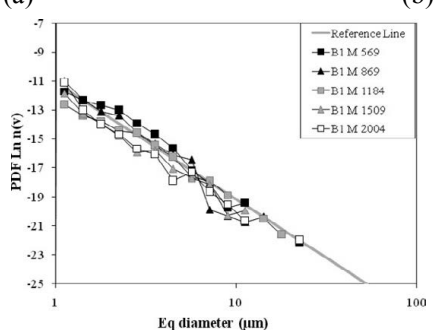

(c)

Fig. 10. Inclusion population density function (PDF) plots for the initial heat B1 during casting: (a) tundish shroud (b) tundish well and (c) mould. The legends used in this figure are the same as in Fig. 9. The values in legends represent the time (sec) after opening of ladle. 


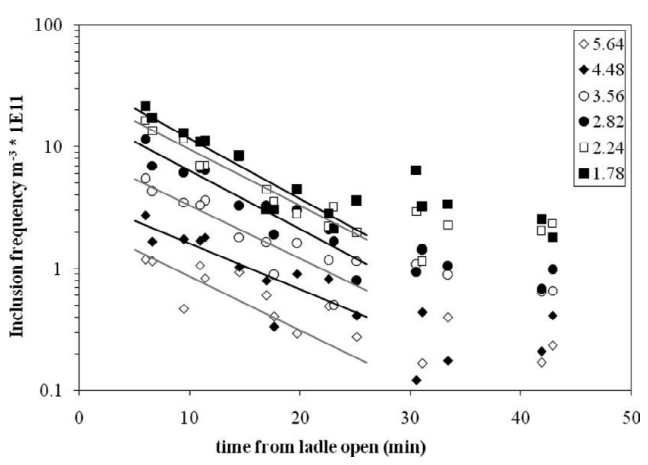

Fig. 11. Time series analysis of the evolution of absolute inclusion frequencies in heat B1. Plotted are the number frequencies $\left(\mathrm{m}^{-3}\right)$ recalculated by integration of the inclusion PDF in Fig. 10 per size bin. Values in legends are the equivalent diameter $(\mu \mathrm{m})$ of each inclusion groups (size bin).

idation will cease as the steel flow volume becomes shielded from oxygen sources. Therefore, the inclusion concentration formed in the early stage of the casting process can be diluted (reduced) by clean steel from ladle with casting time, and eventually be leveled out at the comparatively low concentration of inclusions in ladle steel.

That is, the initial batch of new alumina that has been formed at ladle-open time was instantly well-mixed throughout the caster system, and is then gradually flushed out by a cleaner steel input flow, which can be simulated by the classical Continually Stirred Tank Reactor (CSTR) models in chemical engineering, We have fitted such a CSTR tundish model to the log-linear decay part of the inclusion concentrations (lines in Fig. 11). Fitting each size cohort separately, we obtain replenishment frequencies of $\mathrm{Q} / \mathrm{V}$ $($ decay constant $)=0.10-0.11$ for the size cohorts $(2-6 \mu \mathrm{m})$ that show pronounced time-decaying PDF curvature, perfectly consistent with the known dimensions of the system. This explanation is also consistent with the time variation of [N] content in tundish in Fig. 10. We conclude that batch formation of new alumina inclusions due to tundish-initiation reoxidation, followed by passive flushing by a clean-steel stream from the ladle, is sufficient to explain the observed inclusion behaviour. Specifically, although intra-tundish flotation of inclusions, or inclusion loss to sidewall attachment cannot be neglected, the main factor of the cleaning of inclusion can be explained by this simple CSTR model.

\section{Summary of Changes in Inclusion PDF from Second- ary Steelmaking to Casting}

Summarizing over the entire dataset acquired for the inclusion behaviour in Ti-alloyed Al-killed steel in two series A and B, the inclusion chemistry is invariably strongly dominated by alumina. This is also true for many other Al killed steels, and often any secondary phases or oxide compounds are relegated to extremely minor amounts. In this case, significant process information can still be obtained from inclusion size distributions. Size distribution analysis requires measurement of large numbers of inclusions. In order to obtain statistically reliable inclusion distribution, from our experience, at least several hundreds of inclusions should be analyzed. In these days, many steel producers employ automated analysis techniques, such as SEM AIA in this study and OES(Optical Emission Spectrometry) PDA at Posco to quantify both size and chemical composition of inclusions. However, the characterized results are typically plotted as histograms with arbitrary bin spacings. In such a case, the results of size distribution cannot give the quantified PDF profile and can mislead the interpretation. Only when the inclusion PDF is properly plotted, the analysis can give meaningful information. For example, in the present study, the reoxidation events during the secondary steelmaking process can be accurately correlated with changes in the inclusion PDF.

In order to obtain the inclusion PDF plots representing the secondary steelmaking condition, the secondary inclusions forming during the cooling or reoxidation of steel in sampling process should be minimized, or if necessary removed afterward based on logical approach. In the present study, TiN was considered as secondary inclusion because this is not a liquidus-near phase in low-nitrogen Ti alloyed steel. Further, in order to improve the PDF analysis, the sampling technique should be well-established to minimize the formation of secondary inclusions or reoxidation of steel during the sampling itself. Recently, Zhang et al. ${ }^{17)}$ shows the importance of the sampler design to minimize reoxidation of steel inside the samplers to keep the original inclusion distribution.

The inclusion PDF plots from secondary steelmaking conditions display two basic size distribution shapes: purely or in combination of (i) a lognormal particle size distribution and (ii) power law distribution. A lognormal shape PDF appears whenever evidences indicated the occurrence of active nucleation (deoxidation or reoxidation) in the present study, which is supported by the modeling of nucleation and growth kinetics. $^{7,8,18-20)}$ This situation is characterized by net mass transfer between solute and inclusion. Inclusion PDF show a power-law distribution that is linear on the logarithm PDF plots when there is no deoxidation or reoxidation event for a while in the secondary steelmaking, which is expected for the processes where particle breakup/coalescence control the size distribution (e.g. Refs. 13, 21 and 22). This situation is characterized by chemical equilibrium between solute and inclusion and no net mass transfer.

Monitoring the inclusion size distribution for the entire course of the secondary steelmaking process can be very useful to identify the unexpected oxidation or reoxidation event and improve the melt cleanliness. Although a stirring process with Ar gas is generally thought to be helpful to reduce the non-metallic inclusions, we found no noticeable improvement in melt cleanliness in some of the heats investigated (see Fig. 8), but instead the gas purging appeared to induce reoxidation in at least one case. The inclusion PDF can be used similarly for many other cases.

Although Ti-alloyed steel is the object of this study, a majority of inclusions is pure alumina inclusions, like many other Al killed steels. Surprisingly, the power law distributions of the inclusions in the present study always show a slope exponent of -3.5 as shown in Eq. (8) regardless of the processing units (for example, RH-OB degasser, stirring station, tundish or mould). This leads to the generation of the Reference Line distribution for this specific plant layout, as defined in Eq. (8). It should be noted that the derived exponent is very close to the value of -3.3 predicted from numerical simulation studies ${ }^{7,8)}$ of $\mathrm{Al}_{2} \mathrm{O}_{3}$ inclusions. Based on this concurrence of predictions and observations, it is possible to propose a general Reference Size Distribution of $\mathrm{Al}_{2} \mathrm{O}_{3}$ inclusion after enough time for mixing in Al-killed steel melts:

$$
f(r)=a \cdot r^{-3.5}
$$

where $f(r)$ is the population density function of $\mathrm{Al}_{2} \mathrm{O}_{3}$ inclu- 
sions, $r$ is the equivalent diameter of the inclusions, and $a$ is the pre-factor which may be varied with degree of initial deoxidation. The pre-factor $a$ can be also changed with processing time and processing condition due to the flotation removal of inclusions. This distribution function will be very valuable for practical steelmaking application. For example, once the PDF of one specific bin size (for example, $1-2 \mu \mathrm{m})$ is determined by the inclusion analysis, the pre-factor $a$ in Eq. (9) can be determined and in turn can predict the inclusion population distribution of entire range of inclusion sizes.

The inclusion PDF with exponent of -3.5 in Eq. (9) can be applied to the $\mathrm{Al}_{2} \mathrm{O}_{3}$ inclusions. Then we can question about the PDF of other types of inclusions such as $\mathrm{MnO}-$ $\mathrm{SiO}_{2}\left(-\mathrm{Al}_{2} \mathrm{O}_{3}\right)$ type after $\mathrm{Mn} / \mathrm{Si}$ deoxidation and $\mathrm{CaO}-\mathrm{Al}_{2} \mathrm{O}_{3}$ type after $\mathrm{Ca}$ treated-Al deoxidation. Although it is not wellobserved or simulated yet, it may be possible to have the same exponent as $\mathrm{Al}_{2} \mathrm{O}_{3}$ inclusion of the present study. It is believed that the most important factor controlling the diffusion growth of inclusion be the interfacial energy of inclusion in molten steel; the shape change of lognormal type distribution (shape parameter of lognormal function, $b$ in Eq. (6)) is highly dependent on the interfacial energy as discussed by Van Ende et al. ${ }^{6)}$ However, when the inclusion size distribution is controlled by the physical collision/ breakup, the PDF may be less dependent on the interfacial energy but more dependent on the probability of the collision in molten steel itself which is governed by fluid dynamics. This should be investigated further. If this is the case, the only effective way to modify the inclusion size distribution may be the change of fluid flow pattern in molten steel to enhance the collision and floatation. If this is not the case, the modification of interfacial energy of inclusions by addition of dilute elements like surface active elements can be applicable to the active modification of the inclusion size distribution.

\section{Summary}

In the present study, the evolution of non-metallic inclusions in the series of Ti-alloyed Al-killed steel samples during the entire secondary steelmaking and casting processes was investigated using the automated inclusion analysis (AIA) implemented with the aid of SEM-EDS technology. An inclusion analysis technique using the EDS spectrum and a data conversion process to inclusion population density function (PDF) were introduced. The inclusion PDF plots with equivalent inclusion size show either the lognormal type function or the power law type function or a combination of them (Strictly speaking, the PDF should be combination of the two type functions to a certain extent). From the inclusion PDF plots, the followings were concluded:

(1) Inclusion PDF shows the lognormal shape function just after the nucleation/growth event such as deoxidation or reoxidation. Then, the lognormal shape PDF changes to the power law shape function within a rather short mixing time (less than $10 \mathrm{~min}$ ).

(2) The power law type inclusion PDF of $\mathrm{Al}_{2} \mathrm{O}_{3}$ inclu- sions always shows a similar PDF regardless of process condition during the secondary steelmaking: $f(r)=a \cdot r^{-3.5}$ where $a$ is the pre-factor and $r$ the equivalent radius of inclusion.

(3) Inclusion PDFs of the examined series show that stirring with Ar gas bubbling may induce an unexpected reoxidation of steel, in this case possibly due to the open eye formation.

(4) When a series of heats are being cast, it is found that a large amount of inclusions can be generated in tundish during the discharging of the first heat of the series from ladle to tundish. However, the steel cleanliness can become improved and constant after about 20-25 min of the casting start of the first heat.

\section{Acknowledgements}

We want to express our gratitude for stimulating discussion to a large number of colleagues at Tata Steel Europe, especially to A. Kamperman, S. van der Laan, M. Koolwijk, G. Abbel and J. Small. Many people aided with critical technical support, especially F. Grimberg, F. van der Does, E. Bouwens and others. We express our gratitude to Tata Steel Europe, and the BOS no. 2 plant at IJmuiden Works for support of the study and permission to publish these results. We also thank L. Zhang, and M.-A. Van Ende for in-depth discussions. M. Higgins is thanked for permission to use his stereological size data correction program, CSD Corrections.

\section{REFERENCES}

1) M. Wang, Y. Bao, H. Cui, H. Wu and W. Wu: ISIJ Int., 50 (2010), 1606.

2) C. Wang, N. Verma, Y. Kwon, Y. W. Tiekink, N. Kikuchi and S. Sridhar: ISIJ Int., 51 (2011), 375.

3) S. Basu, S. K. Choudhary and N. U. Girase: ISIJ Int., 44 (2004), 1653.

4) D.-C. Park, I.-H. Jung, P. C. H. Rhee and H.-G. Lee: ISIJ Int., 44 (2004), 1669.

5) W.-C. Doo, D.-Y. Kim, S.-C. Kang and K.-W. Yi: Met. Mater.-Int., 13 (2007), 249.

6) M.-A. Van Ende, M. Guo, E. Zinngrebe, B. Blanpain and I.-H. Jung: unpublished works (2011).

7) L. Zhang and W. Plushkell: Ironmaking Steelmaking, 30 (2003), 106.

8) Y. Kwon, J. Zhang and H. Lee: ISIJ Int., 48 (2008), 891

9) A. Randolph and M. Larson, Theory of Particulate Processes. 2nd ed., Academic Press, New York, (1988).

10) Ramkrishna, Population Balance. Theory and Application to particulate systems in Engineering. Academic Press, New York, (2000).

11) M. Higgins: Amer. Mineralogist, 85 (2000), 1105

12) M. Higgins: Amer. Mineralogist, 87 (2002), 171

13) I. Bindeman: Amer. Mineralogist, 90 (2005), 1801

14) M. A. Van Ende, M. Guo, E. Zinngrebe, R. Dekkers, J. Proost, B. Blanpain and P. Wollants: Ironmaking Steelmaking, 36 (2009), 201.

15) I.-H. Jung, G. Eriksson, P. Wu and A. Pelton: ISIJ Int., 49 (2009), 1290 .

16) C. W. Bale, E. Bélisle, P. Chartrand, S. Decterov, G. Eriksson, K. Hack, I.-H. Jung, Y.-B. Kang, J. Melançon, A. D. Pelton, C. Robelin and S. Petersen: Calphad, 33 (2009), 295.

17) Z. Zhang, A. Tilliander and P. G. Jonsson: Steel Res. Int., 81 (2010), 749.

18) B. McCoy: J. Colloid Interface Sci., 240 (2001), 139

19) D. Eberl, A. Drits and J. Srodon: Am. J. Sci., 298 (1998), 499

20) L. Kampmann and M. Kahlweit: Berich. Bunsen-Gesell., 74 (1970), 456

21) Q. Jian and B. Logan: Environ. Sci. Technol., 25 (1991), 2031

22) T. Nakaoka, S. Taniguchi, K. Matsumoto and S. T. Johansen: ISIJ Int., 41 (2001), 1103 